\title{
Hutchinson-Gilford progeria syndrome accompanied by severe skeletal abnormalities in two Chinese siblings: two case reports
}

\author{
Zhimin Xiong ${ }^{1,2}$, Yanmei Lu', Jinjie Xue ${ }^{1}$, Sanchuan Luo ${ }^{1}$, Xiaojuan Xu' ${ }^{1}$ Lusi Zhang ${ }^{1}$, Hao Peng ${ }^{1}$, Wei Li ${ }^{1}$, \\ Dengming Chen ${ }^{4}$, Zhengmao Hu${ }^{1}$ and Kun Xia ${ }^{1,2,3^{*}}$
}

\begin{abstract}
Introduction: Hutchinson-Gilford progeria syndrome is a rare pediatric genetic syndrome with an incidence of one per eight million live births. The disorder is characterized by premature aging, generally leading to death due to myocardial infarction or stroke at approximately 13.4 years of age. The genetic diagnosis and special clinical manifestation in two Han Chinese siblings observed at our clinic for genetic counseling are described in this report. We screened the LMNA gene in these two siblings as well as in their unaffected parents. A homozygous mutation R527C was identified in the affected siblings, and both parents were heterozygous for this variant.

Case presentation: In case 1, the elder 10-year-old female sibling showed the classic physical and radiological changes of Hutchinson-Gilford progeria syndrome in addition to a considerable overlap with the phenotype of mandibuloacral dysplasia.

In case 2, the younger male sibling had begun to show some early physical changes at age six months.

Conclusion: The phenotypic findings in the patients we describe here widen the clinical spectrum of Hutchinson-Gilford progeria syndrome symptoms, providing further recognition of the phenotypic range of LMNA-associated diseases.
\end{abstract}

\section{Introduction}

Hutchinson-Gilford progeria syndrome (HGPS; OMIM: 176670) is an extremely rare but devastating disorder characterized by extremely short stature, low body weight, early hair loss, lipodystrophy, scleroderma, decreased joint mobility, osteolysis and facial features that resemble those of aged persons [1,2]. The incidence of HGPS is estimated to be one in every eight million live births [2]. The diagnosis is usually made by two years of age. About 144 cases have been described in the literature worldwide [1,3]. The pattern of inheritance is uncertain, though both autosomal dominant and autosomal recessive modes have been proposed $[4,5]$.

Recent genetic advances have identified LMNA as a causative gene of HGPS. LMNA encodes lamins A and

\footnotetext{
* Correspondence: xiakun@sklmg.edu.cn

'State Key Laboratory of Medical Genetics, Central South University,

Changsha, Hunan, China

${ }^{2}$ School of Biological Science and Technology, Central South University,

Changsha, Hunan, China

Full list of author information is available at the end of the article
}

$\mathrm{C}$, which are the main components of intermediate filamentous lamina, function as a structural support, and are essential for DNA replication and mRNA transcription. In addition, these proteins play a role in gene regulation and many signal transduction pathways [6]. Here, we report the case of two Han Chinese siblings who harbor a homozygous change that predicts an R527C mutation, similar to that in another case that has been recently reported [3].

\section{Case presentations \\ Case1}

Our patient was a 10-year-old Han Chinese girl, observed at our clinic for genetic counseling, who was the product of an unremarkable pregnancy and delivery. She did not exhibit any abnormal symptoms until the age of five months, when she developed skin thickening over the extensor surface of her joints followed by progressive difficulty in extending her fingers. Shortly thereafter, she began to lose her hair. Her younger brother has a physical appearance similar to his sister. The living blood

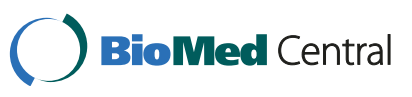


relatives of the parents are negative for the phenotype, and the parents have no blood relationship.

On examination, the girl was found to have an extremely short stature (height $93.5 \mathrm{~cm}$; weight $6.5 \mathrm{~kg}$ ) and a head circumference of $47.3 \mathrm{~cm}$. She was bald, had no eyebrows or eyelashes, and had prominent scalp veins. She also had prominent eyes, a small face and large head, a convex nasal ridge, micrognathia and crowded teeth, narrow shoulders and a horse-riding stance, and a high-pitched voice. There was a generalized paucity of subcutaneous fat. Her atrophic skin was thin, dry and taut, and was marked with diffusely scattered hyperpigmented foci. There was a fixed flexion deformity in all of her fingers. Her anterior fontanel was open $(1.5 \mathrm{~cm} \times 1.5 \mathrm{~cm})$.

Radiography revealed generalized and marked osteoporosis with relative expansion of the metaphyseal areas of her bones, a large cranium and a relatively small viscerocranium. Both her maxillae and mandibles were hypoplastic with crowded teeth, malocclusion and protrusion of her upper teeth. Both of her clavicles were completely absent, the bilateral posterior segments of her first to fourth ribs were partially osteolytic, and the costal head of her right $12^{\text {th }}$ rib was absent. She had severe scoliotic deformities: her cervical segment showed fixed flexion deformities and her fifth lumbar segment had a slightly forward olisthe. There was complete osteolysis and resorption of the superior fragment of both radii, the inferior segment of her right ulnoradial joint had a dislocation, and there was an old, healed fracture of the inside of her right ulna. There were fixed flexion deformities of her phalanges: the middle phalanges of her fingers became more slender, and the distal phalanges of the second to fifth fingers were absent (Figure 1A-C).

Magnetic resonance imaging revealed normal brain tissue with mastoiditis of both middle ears. Our patient had a small pituitary gland with a concavity of the superior border (height about $1 \mathrm{~mm}$. Figure 1D). Audiologic testing revealed conductive hearing loss in the lowfrequency range $(250 \mathrm{~Hz}$ to $500 \mathrm{~Hz})$ in both ears. Echocardiography revealed left ventricular hypertrophy with a mild regurgitation of the tricuspid valve and pulmonary valve (Figure 2D). Carotid ultrasonography detected a narrow carotid artery intima-media thickness.

Laboratory results of hepatic function and her levels of blood calcium, blood phosphonium, blood free triiodothyronine, free thyroxin, thyroid-stimulating hormone, serum cholesterol, triglyceride, lipoproteins, fast
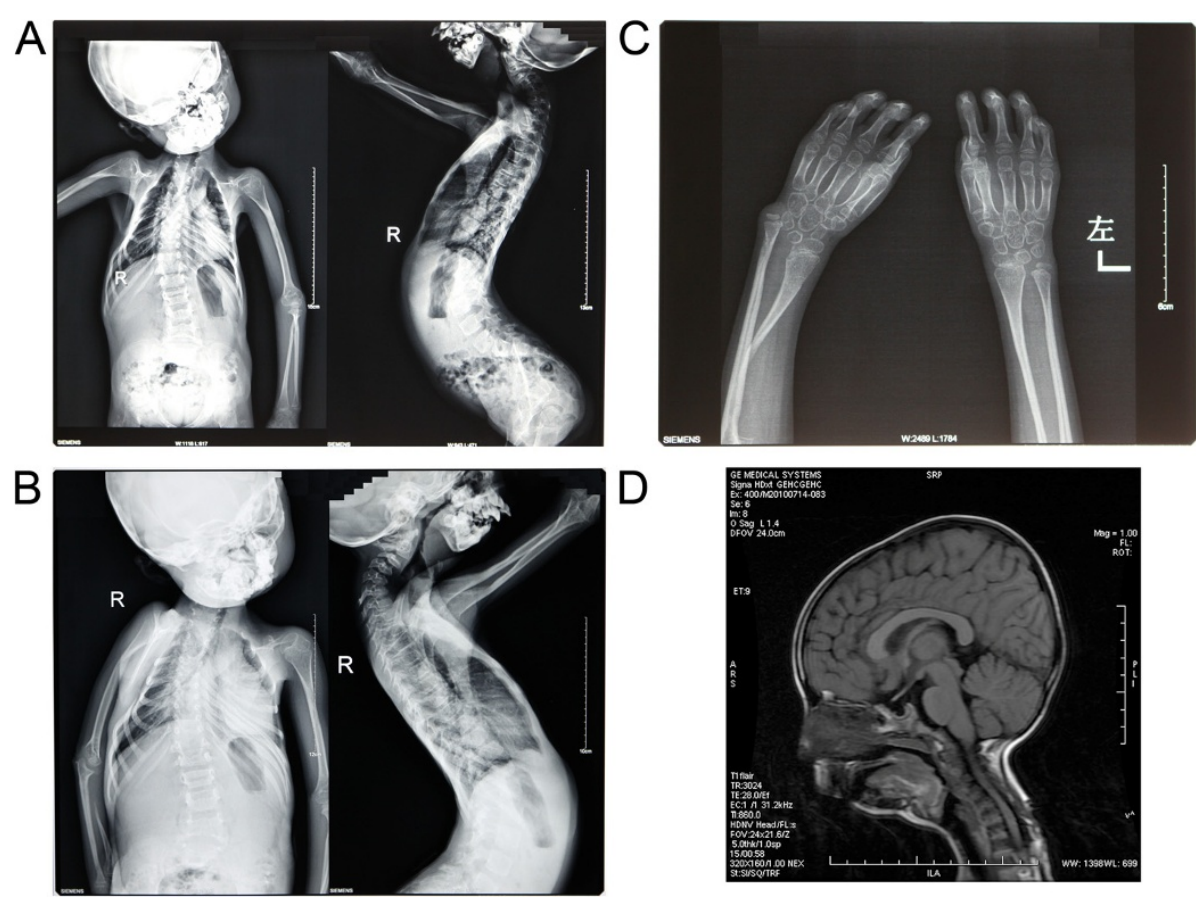

Figure 1 Radiography and magnetic resonance imaging features in case $\mathbf{1}$. (A,B) The elder sibling showed generalized and marked osteoporosis with relative expansion of the metaphyseal areas of the bones, completely absent clavicles, partial osteolysis of the bilateral posterior segments of the first to fourth ribs, absence of the costal head of the right 12th rib; severescoliotic deformities, complete osteolysis and resorption of the superior fragment of both radii, a dislocation of the inferior segment of the right ulnoradial joint, and an old, healed fracture of the interior segment of the right ulna. (C) Fixed flexion deformities of the phalanges in the elder sibling with absence of the distal phalanges of the second to fifth fingers. (D) Magnetic resonance imaging features of the elder sibling showing a small pituitary gland with a concavity of the superior border (with a height of about $1 \mathrm{~mm}$ ). 


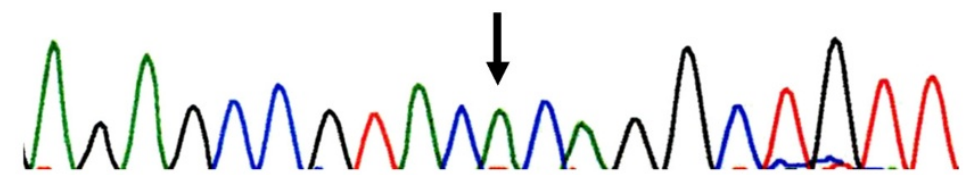

B A G A G C C G T A C G C A G G C T G T T
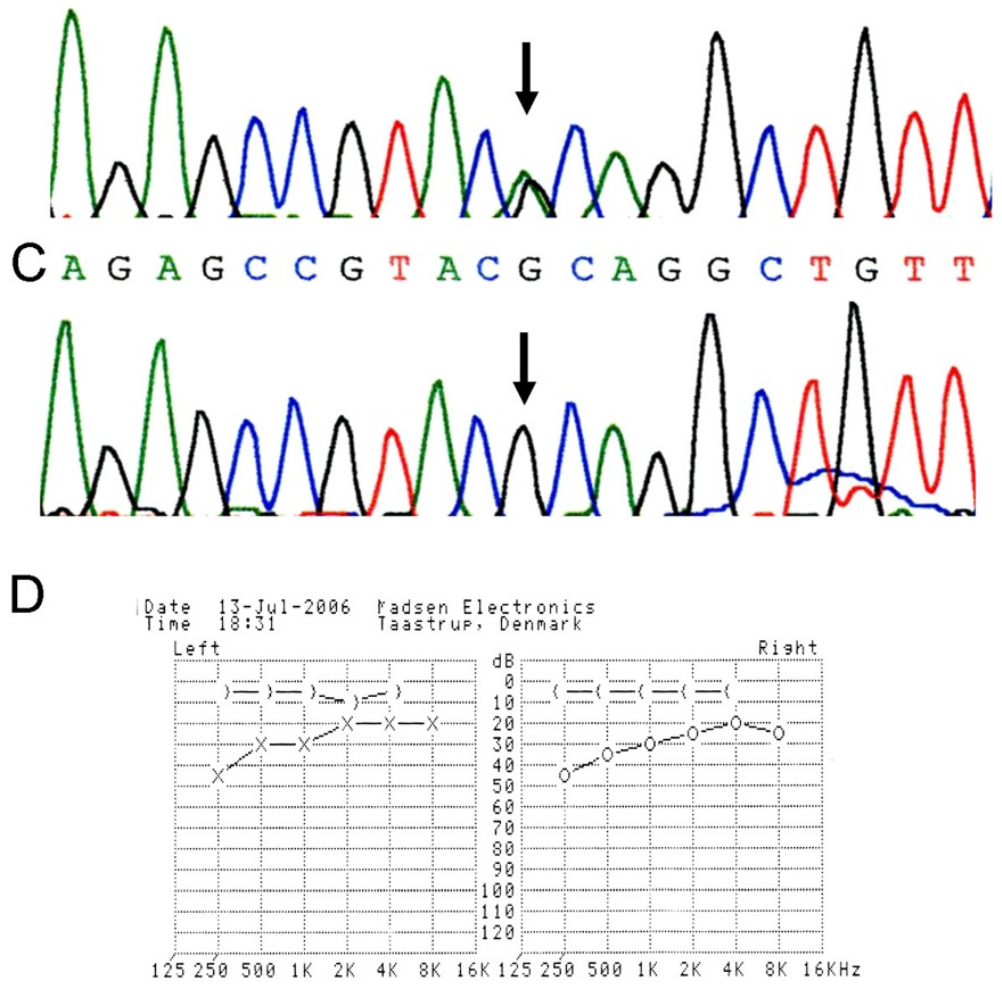

Figure 2 LMNA R527C in the two siblings and their parents and audiologic testing in case 1. (A) Homozygous mutation of R527C in the two siblings. (B) Heterozygous mutation of R527C in parents. (C) Normal control sequence. (D) Audiologic testing showing that the elder sibling had a conductive hearing loss in the low-frequency range $(250 \mathrm{~Hz}$ to $500 \mathrm{~Hz})$ in both ears.

blood glucose, prothrombin time and hemoglobin were all within the normal ranges. Her renal function showed a low level of creatinine $(29.6 \mu \mathrm{mol} / \mathrm{L}$; normal range 53 to $132.6 \mu \mathrm{mol} / \mathrm{L})$. Her platelet count was elevated.

\section{Case 2}

The 18-month-old younger brother of our first patient had begun to lose his hair at the age of six months. He had prominent eyes and open fontanels. His scalp veins were prominently visible. His skin was thickened and showed a striking resemblance to that of his sister. Radiography revealed an attenuation of the skull board, widely patent sutures, and the absence of left and right lateral clavicles.

A chromosome analysis (resolution, 500 to 600 bands) revealed that the siblings had a normal karyotype ((46, $\mathrm{XX})$ and $(46, \mathrm{XY})$ respectively). We then sequenced the entire coding region of the $L M N A$ gene for the affected siblings and their unaffected parents. Mutation analysis revealed a homozygous mutation $1579 \mathrm{C}>\mathrm{T}$, which predicts $\mathrm{R} 527 \mathrm{C}$, in the two siblings; both their parents were heterozygous for this variant. By contrast, no variants of this specific mutation were found in the 100 unrelated control individuals (Figure 2A-C).

\section{Discussion}

Our patient in case 1 exhibited the typical phenotype of HGPS, showing the initial symptoms in the first year of life, severe growth deficiency, expressed lipodystrophy, expressed hair loss, and severe osteolysis of the acra, clavicles, mandible and viscerocranium. However, her severe skeletal abnormalities, which were similar to those of other sibling sets described by Monu et al. in 1990 [7], have a considerable overlap with the phenotype of 
mandibuloacral dysplasia, a disorder mainly characterized by skeletal malformations. In addition, there were other unusual features in our patient. First, the scoliotic deformities, such as the olisthy of her lumbar segments, were much more severe than in the previously reported cases. Second, the degree of osteolysis and resorption of her ribs was much greater. Third, the dislocation of the inferior segment of her right ulnoradial joint and the low level of creatinine that we observed in this patient has, to the best of our knowledge, not been reported before. Fourth, the results of the magnetic resonance imaging revealed a pituitary gland small for our patient's age.

The mode of inheritance of the disease remains largely speculative for now because the patients affected cannot reproduce. Although recurrent heterozygous de novo mutations of LMNA strongly support a mode of inheritance of HGPS as a sporadic dominant disorder, autosomal recessive modes of inheritance have been proposed in siblings and blood relatives [5].

Most of the classic cases of HGPS are reported to be caused by a single base substitution $1824 \mathrm{C}>\mathrm{T}$ in $L M N A$, which activates a cryptic splicing site [8]. Here we identified a homozygous mutation c. $1579 \mathrm{C}>\mathrm{T} L M N A$ in two siblings, which predicts an R527C mutation. Results of the clinical examination of the parents, who were heterozygous for this mutation, were normal. We conclude that the inheritance of the phenotype in our patients is autosomal recessive. The R527C mutation was first described in a 28 -year-old female compound heterozygote patient with $\mathrm{R} 471 \mathrm{C}$, affected with mandibuloacral dysplasia [9]. The homozygous mutation R527C has been reported recently in two Chinese siblings [3] whose phenotype was categorized as atypical HGPS with severe skeletal abnormalities.

Mutations in LMNA have been identified as the cause of at least 12 different inherited disorders [10,11], known as laminopathies, which have significant phenotypic overlap [12]. However, it remains unclear how different mutations in the LMNA gene cause such a variety of phenotypes. For instance, at the same coding site 527, three different substitutions have been reported to cause three different diseases: R527P causes autosomal dominant Emery-Dreifuss muscular dystrophy [13], whereas $\mathrm{R} 527 \mathrm{H}$ is associated with mandibuloacral dysplasia [14], as is the compound heterozygote R527C/R471C [9]. The homozygote R527C causes either atypical [3] or typical HGPS.

The encoding products of LMNA are part of an intermediate filament family of proteins that are composed of an N-terminal end, a coiled-coil rod domain and a globular C-terminal tail domain [15]. The R527C mutation described here resides in the $\mathrm{C}$-terminal tail domain of the protein. The result of the substitution of a basic amino acid (arginine) to a neutral one (cysteine) in this location would disrupt the surface structure of lamins, thus causing the severe diseases observed.

\section{Conclusion}

The phenotypic findings in the patients we described here widen the clinical spectrum of HGPS symptoms, and provide further recognition of the phenotypic range of $L M N A$-associated diseases.

\section{Consent}

Written informed consent was obtained from the patients' mother for publication of this case series and accompanying images. A copy of the written consent is available for review by the Editor-in-Chief of this journal.

\section{Competing interest}

The authors declare that they have no competing interests.

\section{Authors' contributions}

XZM analyzed and interpreted the patient data and was a major contributor in writing the manuscript. XJJ and CDM interpreted the patient data regarding $X$-ray and magnetic resonance imaging. LYM and LSC performed the genetic analysis. XXJ, ZLS, PH, LW and HZM performed laboratory examinations. XK communicated with the patients and revised the manuscript. All authors read and approved the final manuscript.

\section{Acknowledgements}

This work was supported by the National Basic Research Program of China (2012CB517900) and the National Natural Science Foundation of China (81161120544).

\section{Author details}

'State Key Laboratory of Medical Genetics, Central South University, Changsha, Hunan, China. ${ }^{2}$ School of Biological Science and Technology, Central South University, Changsha, Hunan, China. ${ }^{3}$ Key Laboratory of Medical Information Research, Central South University, Changsha, Hunan, China. ${ }^{4}$ Department of Radiology, Xiangya Hospital, Central South University, Changsha, Hunan, China.

Received: 13 August 2012 Accepted: 28 January 2013

Published: 8 March 2013

\section{References}

1. Hennekam RCM: Hutchinson-Gifford Progeria syndrome: review of the phenotype. Am J Med Genet A 2006, 140A:2603-2624.

2. DeBusk FL: The Hutchinson-Gilford progeria syndrome. Report of 4 cases and review of the literature. J Pediatr 1972, 80:697-724.

3. Liang L, Zhang H, Gu X: Homozygous LMNA mutation R527C in atypical Hutchinson-Gilford progeria syndrome: evidence for autosomal recessive inheritance. Acta Paediatr 2009, 98:1365-1368.

4. Beauregard S, Gilchrest BA: Syndromes of premature aging. Dermatol Clin 1987, 5:109-121.

5. Khalifa MM: Hutchinson-Gilford progeria syndrome: report of a Libyan family and evidence of autosomal recessive inheritance. Clin Genet 1989, 35:125-132.

6. Shumaker DK, Kuczmarski ER, Goldman RD: The nucleoskeleton: lamins and actin are major players in essential nuclear functions. CurrOpin Cell Biol 2003, 15:358-366.

7. Monu JU, Benka-Coker LB, Fatunde Y: Hutchinson-Gilford progeria syndrome in siblings. Report of three new cases. Skeletal Radiol 1990, 19:585-590.

8. Eriksson M, Brown WT, Gordon LB, Glynn MW, Singer J, Scott L, Erdos MR, Robbins CM, Moses TY, Berglund P, Dutra A, Pak E, Durkin S, Csoka AB, Boehnke M, Glover TW, Collins FS: Recurrent de novo point mutations in lamin A cause Hutchinson-Gilford progeria syndrome. Nature 2003, 423:293-298. 
9. Pollex RL, Hegele RA: Hutchinson-Gilford progeria syndrome. Clin Genet 2004, 66:375-381.

10. Garg A, Speckman RA, Bowcock AM: Multisystem dystrophy syndrome due to novel missense mutations in the amino-terminal head and alphahelical rod domains of the lamin A/C gene. Am J Med 2002, 112:549-555.

11. Broers JL, Hutchison CJ, Ramaekers FC: Laminopathies. J Pathol 2004, 204:478-488.

12. Mounkes L, Kozlov S, Burke B, Stewart CL: The laminopathies: nuclear structure meets disease. CurrOpin Genet Dev 2003, 13:223-230.

13. Bonne G, Di Barletta MR, Varnous S, Becane HM, Hammouda EH, Merlini L, Muntoni F, Greenberg CR, Gary F, Urtizberea JA, Duboc D, Fardeau M, Toniolo D, Schwartz K: Mutations in the gene encoding lamin A/C cause autosomal dominant Emery-Dreifuss muscular dystrophy. Nat Genet 1999, 21:285-288.

14. Novelli G, Muchir A, Sangiuolo F, Helbling-Leclerc A, D'Apice MR, Massart C, Capon F, Sbraccia P, Federici M, Lauro R, Tudisco C, Pallotta R, Scarano G, Dallapiccola B, Merlini L, Bonne G: Mandibuloacral dysplasia is caused by a mutation in LMNA-encoding lamin A/C. Am J Hum Genet 2002, 71:426-431.

15. Stuurman N, Heins S, Aebi U: Nuclear lamins: their structure, assembly, and interactions. J Struct Biol 1998, 122:42-66.

doi:10.1186/1752-1947-7-63

Cite this article as: Xiong et al:: Hutchinson-Gilford progeria syndrome accompanied by severe skeletal abnormalities in two Chinese siblings: two case reports. Journal of Medical Case Reports 2013 7:63.

\section{Submit your next manuscript to BioMed Central and take full advantage of:}

- Convenient online submission

- Thorough peer review

- No space constraints or color figure charges

- Immediate publication on acceptance

- Inclusion in PubMed, CAS, Scopus and Google Scholar

- Research which is freely available for redistribution 